\title{
Evaluation of Biomedical Imaging in Deep Neural Networks
}

\author{
Karthikeyan K \\ Department of Computer Science and Engineering, SNS College of Engineering, India. \\ sns.cse.karthik@gmail.com
}

\author{
Article Info \\ Journal of Biomedical and Sustainable Healthcare Applications (http://anapub.co.ke/journals/jbsha/jbsha.html) \\ Doi: https://doi.org/10.53759/0088/JBSHA202101004 \\ Received 21 August 2020; Revised form 23 September 2020; Accepted 24 October 2020. \\ Available online 05 January 2021. \\ (C)2021 Published by AnaPub Publications.
}

\begin{abstract}
Whereas the historical background of medical field started in 1895 with Roentgen Wilhelm participating in the first x-rays photograph and proceeded through 1913 with the discovery of mammogram and 1927 with first cerebellar echocardiogram, advanced medicine tomography came into focus in the 1950s with the discovery of PET and ultrasonic image processing. The first computed tomography (CT) scanners was created by Hounsfield Godfrey and CoreMark Allanin 1972, while the first commercialized Magnet Resonance Imaging (MRI) scanners were produced by Raymond Dalmatian in 1977. The creation of general methods and terminology of digitized signal and image processing occurred in tandem with the growth of medical imaging technology in the 1970s and beyond, as well as the advent of digital processors. In an examination of biological applications and analysis in the era of big data and deep learning, this article analyzes background and phraseology: Pattern Classification, Artificial Intelligence, Machine Learning, Big Data.
\end{abstract}

Keywords - Pattern Classification, Artificial Intelligence, Machine Learning, Big Data

\section{INTRODUCTION}

The ability to see the human body utilizing a variety of technologies has transformed healthcare in latest generations and keeps expanding at a fast rate. At a wide variety of temporal and spatial scales, heretofore unknown knowledge on biology and illness is being revealed even more than before. While findings and acceptance of methods linked to quantitative and statistical image analysis have trailed behind image capture technologies, these fields have seen a surge in attention and effort in recent times. This distinctive issue aims to outline and illustrate a few of the "hot" new and exciting opportunities in bioimaging and analysis, with the goal of shedding light on where the domain might go in the coming decades, with a particular focus on areas in which electronic designers have indeed been implicated and might conceivably have more impact. Image capture biophysics, image/signal processing, and image recognition, encompassing computer vision and natural language, are all examples of these fields.

Scientists and software engineers have discovered that the information included in medical pictures is usually sparse when examined via image-based vector spaces, which is a prevalent theme in much of this work [1]. This remark has had a profound impact on many people, and it can be found across the pieces we've collected. Secondly, since electron microscopy is one of the major providers of big data, data-based machine learning methodologies (eg deep learning) are attaining traction due to their excellent efficiency. As a result, data-driven methods such as picture reconstructions for creation and machine learning for image processing are progressing quickly. From bacteria living to human health, image science was used to investigate the complexity of biological systems. This aim has pushed medical and biological imaging researchers to create sensing methods that can monitor cellular interactions over a wide variety of temporal and spatial scales in order to investigate the hierarchy of characteristics that emerge from complex evolutionary processes.

Nanotechnology is being pushed into higher - dimensional feature areas in quest of a deeper knowledge and more accurate diagnostic evaluations. J. Duncan works with the Departments of Bioengineering and Radiography \& Medical Screening, Yale University, New Haven where he started with diagnostic imaging methods for imaging and controlling cancer and cardiovascular disease [2]. On November 1st, 2019, the manuscript was received and on December 1st, 2019, it was edited. techniques for revealing the microbiome's institutional effect on wellbeing and illness, configuration of neurocognitive and biotechnology in learning and memory, and intelligent systems in optical system and perception, where human classification technique and prototype human visual methodologies have reached their constraints. Utilizing datadriven methods currently available via a variety of data mining algorithms, the constraints experienced when modelling instrumentation as linear systems may be addressed. However, many people believe that the most effective and reliable models will combine prototype and data-driven methods.

The articles in this compilation include a wide range of issues that we believe are essential for diagnostic applications in the future. These are distributed among 10 distinct groups of writers, as shown below. We attempt establish a case of the "Special Issue" in our "Profiling the Issue" paper by first examining the modern past of term used throughout the areas of massive data, deep learning, and machine learning in the case of clinical photography. Then we'll present and review the 10 papers by categorizing them into two groups: a.) modality-centric picture collection efforts, such as image restoration, and b.) image recognition and image-guided response initiatives. We will wrap things off by highlighting some of the 
submissions' common threads. This paper is organized as follows: Section II focuses a background analysis on machine learning, big data, artificial intelligence and pattern recognition. Section III focuses on the special issue following a review of the 10 studies. Section IV focuses on the cutting-edge theme while Section V concludes the paper.

\section{BACKGROUND ANALYSIS}

Machine Learning, Big Data, Artificial Intelligence And Pattern Recognition

Wilhelm Roentgen took the first X-ray images in 1895, but it wasn't until the 1950s that modern medical imagery began to take shapes with the inventions of PET and ultrasound scanning. Mammogram was invented in 1913, and first cortical angiogram was performed in 1927. Geoffrey Hounsfield (an electronic scientist) and Cormack Allan established the firms CT scanner in 1972 and Damadian Ray created the first Magnetic Resonance Imaging (MRI) scanners in 1977, two important defining events in computerized diagnostic devices [3]. The fundamental techniques and nomenclature of computerized computer vision and image processing developed in parallel with the advancement of diagnostic imaging technologies and the introduction of modern systems in the mid-1960s and beyond.

Digital image and image analysis was mainly created in the 60s at Caltech JPL, Bell and MIT Laboratory, but was most frequently associated with photography and spaceflight. The "Digital Image Processing Technologies" was admitted as a "Inducted Technologies" by the Space Exploration Hall of Fame in 1994, showing one connection between these fields. The development of the terminology "pattern classification" and its relationship to machine learning and artificial intelligence, all of which have an effect on the 10 articles in this Publication, is perhaps the most pertinent to the current subject.

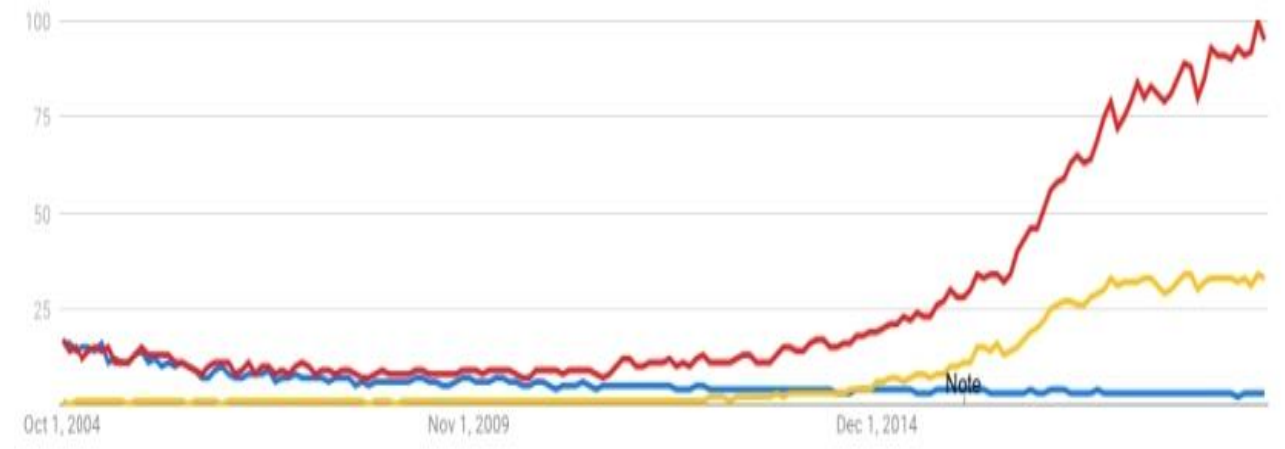

Fig 1. Recognition system (blue) vs. cognitive computing (red) vs. supervised learning (yellow) utilize patterns from Google News from 2004 to 2018.

The ratings on this "compounding interest" line graph represent the term's prominence throughout a certain time period. The relative search traffic for a phrase, as compared to the amount of queries generated by Google, determines Google Trends ratings. The ratings have no numerical value. In same month, two distinct keywords may both have a score of 100 , yet one might get 1,000 search queries while the other gets 1,000,000. Because the scores were scaled from 0 to 100 , this is the case. A value of 100 indicates that the relative search volume is the greatest. These periodic scores are based on the month's overall average everyday search traffic. A falling and rising line does not typically represent a transition in prominence; but instead that overall search usage has risen or dropped significantly (see Fig 1).

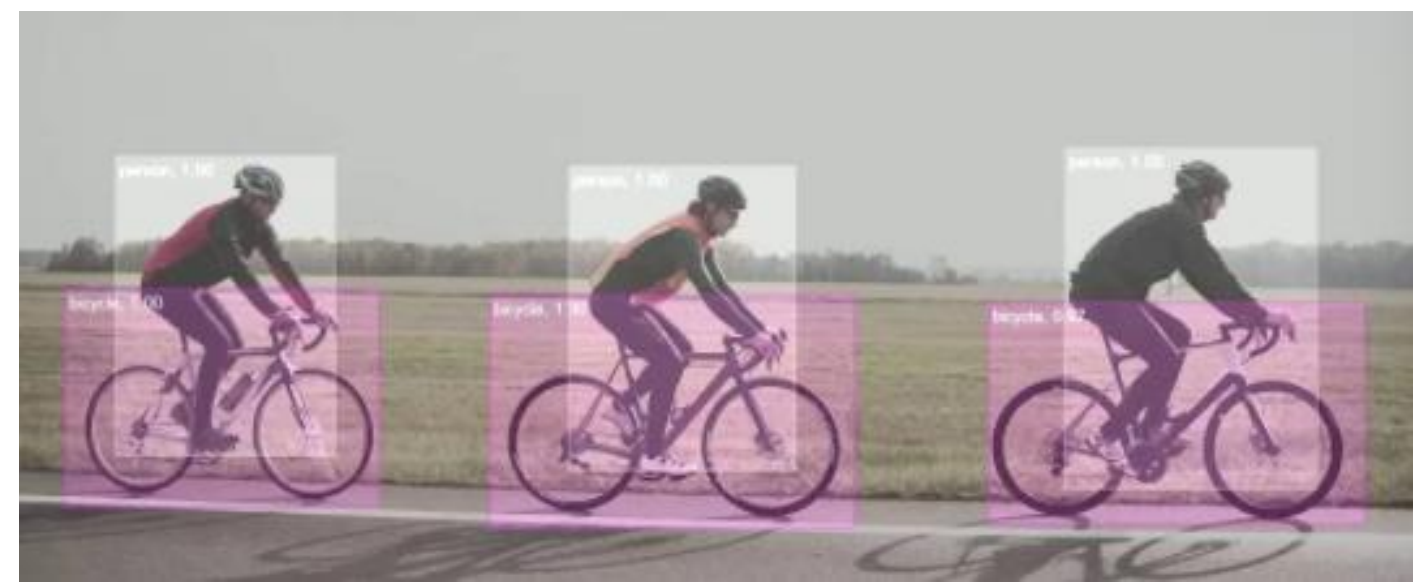

Fig 2. Computer Vision and image processing vision problem that requires classification techniques to solve

Recognition system examines input data for patterns. While exploratory and descriptive classification technique attempts to find patterns in data generally, explanatory pattern recognition begins by classifying the patterns found. As a 
result, classification technique is used to deal with both of these situations, with various supervised classification techniques being used based on the use case and data type [4]. As a result, pattern recognition is more of a collection of frequently unrelated information and methods than a single methodology. Advanced technologies often need the capacity to recognize patterns. Words or phrases, pictures, or audio recordings may all be used as pattern recognition data inputs. As a result, recognition system is more comprehensive than computer vision, which focuses on picture identification (see Fig 2). Pattern recognition, description, categorization, and grouping by machines and computers are significant issues in many technical and scientific fields, including physiology, neurology, psychiatry, advertising, machine learning, and machine learning.

S. Koinuma, Y. Umesono, K. Watanabe and K. Agata in [5] described patterns as "the polar reverse of chaos; it is a loosely defined object that might be officially named." In other terms, a pattern may be any item of relevance that needs to be recognized and identified: it is significant enough that its name should be known (its identity). As a result, patterns may be defined as recurring tendencies in different types of data. Fingerprints picture, a handwritten calligraphy phrase, a reference image, or a voice signal is all examples of patterns (see Fig 3). A pattern may be seen physically, such as in pictures and movies, or statistically, using statistical techniques.
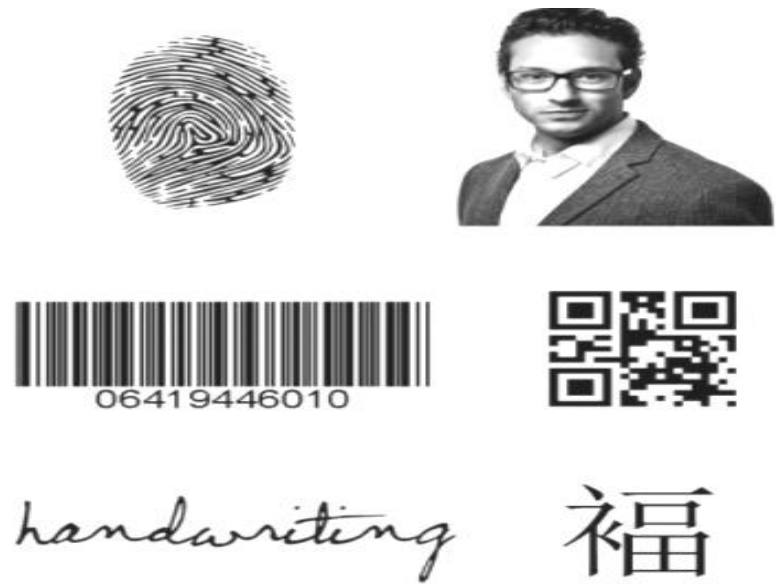

Fig 3. Patterning examples: fingerprints, faces, barcodes, QR codes, handwriting, and character images

One of two activities may be used to recognize and classify a pattern:

- The input sequence is classified as a member of a specified class using classifier. (Informative)

- Unsupervised learning gives a previously unknown class to an input pattern. (Explorative)

Typically, the identification issue is presented as a categorization or classification problem. The classifications are either specified (supervised categorization) by the system or learnt (classification techniques) based on patterns similarities (in unsupervised classification). Potential uses that are not just difficult but also extremely intensive are driving the evolution of pattern classification. Pattern recognition aims to prove that a human being's decision-making process is linked to pattern classification in some way. The next movement in a chess game, for example, is determined by the present pattern on the board, and the decision to purchase or sell shares is based on a complicated pattern of financial data. As a result, the aim of pattern classification is to decipher these complex decision-making systems and to use computers to automate these tasks.

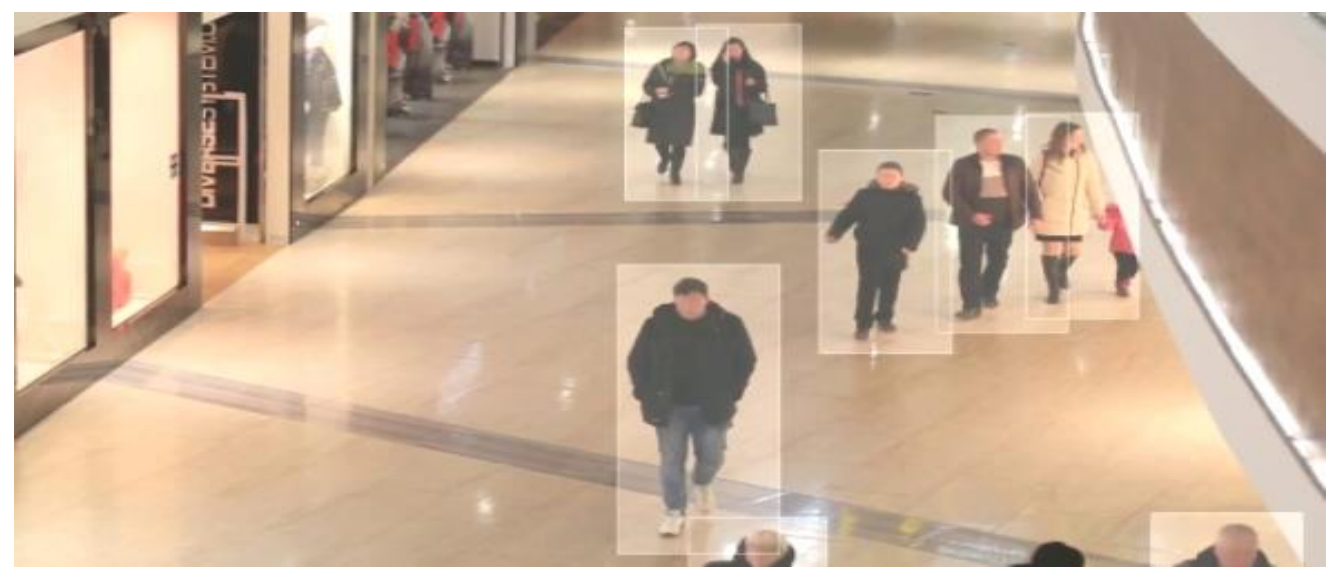

Fig 4. Video deep learning to detect people and recognize them.

Pattern recognition is the examination of how computers can examine their surroundings, learn to identify different patterns and insights from their backgrounds, and make logical judgments about the patterns' categories [6]. Objects are 
allocated to a category during recognition. Because Recognition System is such a dynamic and wide subject, there are many definitions. "A categorization of input data through extraction of significant characteristics from a large amount of noisy data," says one earlier study of pattern classification. This is a scientific field whose goal is to categorize of things into a large number of groups or classes. Most computing technology systems designed for decision-making include classification techniques as well." Pattern recognition is a technique that reduces maps, or labels information. The technique of matching information previously recorded in a database with additional information based on their characteristics is known as pattern classification in computer engineering.

Artificial intelligence (AI) is the emulation of human intellect in which computers are taught to think and behave in the same way as people do. AI disciplines, for example, seek to help computers perform difficult human identification tasks like identifying faces or items. As a result, pattern recognition is considered to be a part of AI.

Pattern recognition and ML are frequently used to build ML techniques that can rapidly and correctly identify and discover unique trends in data in today's era of Digitalization. Pattern recognition can be handy in a variety of situations, including quantitative data evaluation and picture analysis. AI technology is used in the majority of modern pattern recognition applications. Natural language processing, content pattern matching, face detection, motion acknowledgement, recognition for video computational intelligence assessment (see Fig 4), and medical computer vision in health coverage are some of the most commonly used applications.

\section{Pattern Recognition's Advantages}

Depending on the implementation, pattern detection systems offer a variety of advantages. Finding shapes in data can aid in the analysis and prediction of emerging developments, as well as the development of preventive measures based on particular pattern metrics. There are also the following benefits:

- Recognition: Detected trends assist in the identification of objects at various varying distances (for instance, in video-based machine learning) or the recognition of potentially dangerous occurrences. With video deep learning, classification technique is utilized to identify individuals using facial recognition or mobility analysis. New AI algorithms have recently been developed that can recognize individuals based on their stride or walking style. Pattern recognition technologies enable us to "see beyond the box" and identify situations that people might overlook. Over a large quantity of data, algorithm patterns may identify extremely small changes in data or connections between variables. This is critical in medical applications; for instance, deep neural networks are used to make a diagnosis neurological disorders using neuroimaging visuals. Patterns matching using an intrusion detection system (IDS) to analyze network services or assets for fraudulent attacks or policy breaches is a common information processing example in data security and IT.

- Prediction: In several information processing initiatives, such as in trading markets to anticipate share prices and other financial possibilities, or in advertising to identify patterns, predicting data and generating predictions of future events play a significant part. Contemporary machine learning techniques offer highquality knowledge patterns identified in near real-time, which allows for better judgment. This allows for decision-making based on accurate, data-driven information. The speed of current AI computer vision applications, which outperform traditional techniques and allow applications, is a key issue. Medical pattern recognition, for example, can detect predefined criteria in data and provide doctors with critical information quickly.

- Big-data analytics: Artificial neural network enabled the detection of patterns in massive amounts of data. Traditional statistical techniques would not have been able to handle use situations like this. In the medical field, pattern recognition is critical, - particularly for forensic examination and Gene sequencing. It has been used to create vaccinations to combat the COVID-19, for instance.

Recognition system as a topic arose in the mid-1970s, and Duda and Hart's classic book Predictive Modeling, first released in 1973 [7], is arguably the greatest example. This book and topic sprang from the wider subject of Electronic Engineering (EE) as a kind of intelligent (electronics) signal processing techniques, and it became a well-established specialty in the EE education scheme at the moment. In some ways, the goal of analytical thinking was to create algorithms that could be incorporated in hardware and software to undertake smart tasks similar to those performed by humans, such as detecting trends in an electrocardiography or discovering objects in images. In most cases, decision makers extracted features from data, which were subsequently processed using heuristics, logical decision tree, or classifiers evaluations based on The neural positivist paradigm. As the 1980s proceeded, software engineers got more fascinated in the subject, and decision-making algorithms started to employ more and more data in order to need less human input, resulting in the creation of the term "image and video processing." Cognitive science neural pathways, for example, were constructed on layers upon layers of basic decision-making modules loosely patterned after human neural systems. It's intriguing to observe how these terminology developed in the early $21^{\text {st }}$ century and beyond, with detection and recognition becoming less frequent and machine learning and pattern detection becoming even more widespread. The concepts of "Massive Data" and "Artificial Intelligence" are definitely linked to the above, with the latter's use fluctuating in the last half-century, but (in at least a few interpretations) being used to encapsulate anything and everything linked to computer systems and classifiers.

\section{Context of Medical Imaging}

\section{THE SPECIAL ISSUE}

The method and practice of picturing the inside of the body for medical evaluation and clinical interventions, as well as visible depiction of the operation of particular anatomical structures, is known as clinical photography (physiology). Clinical photography is used to reveal internal organs that are hidden beneath the skin and vertebra, or to diagnose and 
treat sickness. Modern medicine generates a record of normal human anatomy and physiology that may be used to discover abnormalities. Despite the fact that the tomography of dissected methods and equipment are conceivable, these procedures are often categorized as diseases instead of diagnostic images. It integrates X-ray radiograph, ultrasonography, magnetic vibration images, elastography, endoscopic.

Other methods that provide data that may be shown as a variable graphs vs. time or map that include information about the measurements sites include magnetoencephalography (MEG), electroencephalography (EEG), electrocardiography (ECG) and others. These techniques may be classified as kinds of clinical photography in another field in a restricted perspective. Globally, 5 billion computed tomography examinations were performed in 2010. Medical imaging sensitivity accounted for over half of all electromagnetic exposure to radiation in the United States in 2006. CMOS electronic circuit slices, power electronics handsets, detectors such as imaging devices (especially CMOS detectors) and bioelectronics, and manufacturers such as embedded systems, micro controllers, computerized sensor manufacturers, internet producers, and system-on-chip gadgets are all used in the production of healthcare visualization devices. Diagnostic imaging chip sales were 46 million units and $\$ 1.1$ billion in 2015 . Clinical imaging is often understood to refer to a set of nonsurgical methods for collecting images of the body's internal architecture. In this restricted sense, imaging may be regarded of as the solution of inverse mathematical issues. This implies that the outcome insinuates the cause (the characteristics of body tissue) (signals that have been observed). Diagnostic ultrasound inquiries are composed of auditory depressurisation and reflections that move through the tissue to expose its structural properties. In projectional radiographs, the scanner releases $\mathrm{X}$-ray radiation, which is gathered at various rates by various kinds of tissue, such as bones, muscles, and adipose.

In the clinical setting, "undetectable illumination" medical imaging is often referred to as "radiography" or "medical imaging," and a radiologist is the medical professional who interprets (and occasionally acquires) the pictures. Medical imaging using "visible light" refers to digitized video or still images that may be seen without the need of specialized equipment. Visible light imaging is used in dermatology and wound treatment, for example. The technological components of medical imaging, especially the capture of medical pictures, are referred to as diagnostic radiography. Although certain radiological procedures are done by radiologists, the radiologist or radiography technician is typically in charge of collecting medical pictures of image quality. Based on the circumstances, clinical imagery is classified as a sub-discipline of bioengineering, clinical physicist, or healthcare. Device, sensing unit (e.g., radiation therapy), designing, and quantitative are typically the domains of bioengineering, healthcare science, and computer programming; investigation into the implementation and explanation of image processing is typically the domain of medical field and the clinical subdiscipline pertinent to the medical problem or area of medical technology (neuroscientific, receiving care and treatment, psychology, cognitive science, and so on) under research. Many clinical imaging methods have industrial and scientific uses as well.

\section{Radiography}

Modern medicine makes use of two types of radiological pictures. Fluoroscopy and projected radiographs are two techniques that may be used to guide a catheter. Notwithstanding the advancement of 3D thermography, these 2D methods are still widely used because of their cheap cost, good clarity, and reduced irradiation doses depending on the specific application. The earliest imaging technology accessible in contemporary medicine, this imaging modality uses a broad beam of $\mathrm{x}$-rays to acquire images.

In a comparable way to radiographic, fluoroscopy creates real-time pictures of inside body systems, but with a steady $\mathrm{x}$-ray input and a lower dosage rate. Vital organs are seen as they operate using contrast media like barite, iodine, and air. When continual feedback during an operation is essential, radiograph is employed in image-guided surgeries. After the radiation passes through the region of interest, it must be converted into a picture using an image sensor. A fluorescing screen was used at first, but this was soon replaced by an Image Amplifier (IA), which was a huge vortex tube with a cesium iodide-coated receiver end and a reflection on the opposing end. A TV camera subsequently took the place of the mirror. X-rays, or projectional radiography, are frequently used to diagnose the kind and severity of fractures as well as to identify pathologic abnormalities in the lungs. They may also be used to see the anatomy of the gastrointestinal tract using radio-opaque contrast fluids, such as barium, which can aid in the diagnosis of ulceration and some kinds of colorectal cancer.

\section{Magnetic Resonance Imaging}

A magnetic resonance imaging (MRI) scanner, also recognized as a "nuclear magnetic resonance imaging (NMR) image processing" detector, uses strong magnets to radicalize and titillate hydrocarbons nucleolus (i.e., solitary particles) of molecules of water in body cells, culminating in a perceptible transmitter that is geographically encrypted and pictures of the skin. A radio frequency (RF) wave is emitted by the MRI equipment at the resonance frequencies of hydrogen bond on molecules of water. The signal is sent to the area of the body being studied through a radio wave antenna (also known as "RF coils"). Atoms absorbed the RF pulse, changing their properties with respect to the fundamental magnetosphere. When the Rf signal is switched off, the particles "loosen" and return to synchronization with the core magnetic, emitting radio signals. The hydroxyl groups on water emit broadcast emissions, which is recorded and reassembled into a picture. The Larmor resonance is based on the degree of external magnetic fields and the chemical nature of the nucleus of relevance. The main field (usually 1.5 to 3 teslas) is used to radicalize the atomic nuclei in MRI; the secondary region (generally 1.5 to 3 teslas) is used to radicalize the atomic nuclei; and the third field (usually 1.5 to 3 teslas) is used to radicalize the atomic nuclei in MRI. a geographically homogenous radio-frequency (RF) signal for manipulating the atomic nuclei to generate quantifiable impulses, gathered using an RF antenna; and slope levels that can be changed to vary in time and space (on the order of $1 \mathrm{kHz}$ ) for spatially coding, often usually called slopes. 
Survey

This problem includes 10 papers from the most prominent scientists in the diagnostic imaging industry who are utilizing quantitative methods to influence their techniques and enhance the usefulness of data included within and generated from clinical pictures, as mentioned above. The issues have been discussed by a diverse group of writers, the majority of them are from different organizations or businesses and are from various parts of the world. The articles are all motivated by applications, yet they demonstrate a wide variety of technological advancements. We believe they may be grouped into two major subgroups, which are divided into the subheadings below: A.) picture capture and creation; B.) image classification and actual image action, involving the incorporation of non-image information like genetics. Absorption and synthesis of images (A) Five distinct groups of authors look at how statistics training influences picture creation in this first part. The publishers of an article titled "Computational Modeling in Ultrasound Scans," written by R. van Sloun, R. Cohen and Y. Eldar in [8], look at how deep, information training can be applied to all elements of ultrasound scans, from concepts at the intersection of raw audio obtaining (such as module facilitates forming) and portrait structure to studying flexural guidelines for color Doppler acquirement to studying clutter inhibition techniques. They provide an intriguing picture of ultrasound's future, based on ultra-portable and sophisticated scanning that enables intelligent, cordless devices for a variety of purposes. The researchers point out that the large amount of information that must be analyzed to apply the current techniques limits the capabilities of computed tomography equipment. Receiving modulation techniques and accompanying signal processing are placed to great strain when high-frame rate 3D positron emission tomography with advanced modulation technique and precise blood- as well as cellular imaging technologies are used, for example. During interactive concentrate, the clustering approach is also utilized to allow fine-scale phases alterations, which limits the sample rate and the absorption degree.

P. Hanrath in [9] propose novel echo model techniques in which duplicates of the audio pulses with uncertain magnitude and durations are used to depict the program's reaction to tissues manual user sparse. This sparse-data paradigm may decrease sample rates considerably without compromising performance. Analog microbeamforming methods, which are currently used to speed up processing at the expense of picture quality, are no longer required with compressed sensing techniques. They demonstrate how effective sampling allows for the incorporation of new computerized imaging methods in a variety of medical sonography applications. In their paper, they also provide a variety of examples, concluding that integrating model- and computation strategies is usually the most effective. The notion that front-end beamforming techniques may be developed using machine studying by understanding the latencies and apodizations by constructing specialized delay layers is one of the most interesting aspects of this research. To translate pre-delayed dataset to framework for combining outcomes, stacking auto - encoder or convolution neural networks may be employed. Similar structures may figure out how to convert unprocessed RF data into B-mode pictures that are optimized. In spectral Doppler applications, deep networks have been and may be used to estimate spectra. Supervised learning and discretization may be utilized to create super resolution ultrasonic, similar to the optical microscope applications mentioned below. Deep networks have been used in clinical echocardiography to help identify optimum perspectives.

In their second piece headlined "Complex Learning feature extraction and improvement in electron microscopy", In [11] provide some summary of efforts to advance the field of computerised microscopes and optic sensing technologies for microscopes utilizing deep learning techniques. They begin by outlining the fundamentals of inverted issues in electron microscopy, then going into how deep learning may be used to solve these issues, usually using supervised techniques. Then they concentrate on applying deep intelligence to these databases in order to achieve single picture hyper magnification and image improvement. They present work that uses various deep neural network designs, such as deep learning approaches, to estimate absent temporal frequency and achieve expanded depth of field (DOF) (GANs). The use of machine learning to confront the restoration of specific molecule alignment pictures at exceedingly fine spectral resolution, recognized as photoactivated localization microscopy (PALM) and variational optical renovation microscopy is possibly one of the most thrilling areas mentioned in this paper (STORM). When supervised learning was used in both instances, the methods were much quicker while retaining the same good image quality. Microscopy is an excellent area for machine learning, according to the scientists, since the observational information are usually collected under highly regulated circumstances, such as constant and repetitive lighting and concentration. According to the researchers, new smart technologies might be designed to perform particular image processing activities by combining the measurement system with a prediction model that could even anticipate which measurement is needed next. Other articles in this issue have come to similar findings on task-specific, customized, "thinking" imaging methods.

The $3^{\text {rd }}$ piece by this team, " PET Deep Learning: from Radiation Identification to Statistical Image Restoration," by [12], looks at how deep learning may be used in PET photography. The researchers talk about how deep learning may be used in multifunctional image analysis using PET, PET-CT, and PET-MRI. They discuss how deep learning affects both the detectors and qualitative picture restoration. Given that genuine collinear count data, dispersed photoelectron occurrences, and additive noise occurrences all contribute roughly the same amount to PET sensing, and given the difficulties postured by low internal sensing efficiency, efficient digital signal of sensor signals is critical in the quest for the best balance of dose distribution, imaging duration, and picture quality. Regarding the dispersion of photoelectric rays captured by photodiodes, detector processing includes calculating the time and location of absorbing events inside each detector crystal. Given the multiple parameters that effect light distribution within a detector, a range of machine learning algorithms have shown promise in boosting sensitivity, ranging from traditional statistical trend detection algorithms to convolutional neural networks (CNNs). Now that quick waveform capacitive sensors are obtainable, classifiers has been used to reliably anticipate the position and commencement time of very intense photons, as the authors highlight in their summary. They also talk through how a variety of statistical techniques and artificial neural implementations are 
increasing the effectiveness of attenuator and scatters correcting techniques, as well as incorporating patient parameter into restricted maximal probabilistic reconstruction.

Machines and data-driven training were utilized to compensate for dispersion and absorption while also decreasing noise in the reconstruction parts of the picture creation methods. New concepts in the area going ahead includes attempting to identify pattern connections amongst higher and lower counts information in an attempt to one day predict high data sets from restricted data sets, as mentioned in citation 166 of their article. In most of the area of clinical imagery, magnetic resonance imaging (MRI) has been the go-to modality. Rather than presenting a comprehensive overview of the field, we encouraged the authors of the $4^{\text {th }}$ piece in this area to focus on one specific aspect, resulting in "Computer Vision for Efficient Magnetic Resonance Fingerprinting (MRF) Cell Structure Quantification" as the title of their work. A single fast MRI capture may generate statistical maps of various tissue characteristics at the same time using this method [13]. To create a thesaurus of waveforms using Bloch equation simulation, the MRF method was originally designed with parameter estimation and regularization in mind. The most recent methods presented in this article, on the other hand, show how deep learning can now speed up the derivation of qualitative representations from MRF data.

The researchers go into detail on how artificial neural network may speed up vocabulary development, which is critical for systems that need to calculate new vocabularies often or measure many tissues characteristics at the same time. They also mention how deep learning may allow for quicker, more reliable patterns identification by skipping vocabulary creation and explicitly estimating tissue property prices from observed information, allowing for quicker, more reliable pattern classification. In essence, modified versions of these methods may be able to produce quicker and more reliable reconstruction of cellular attribute maps, which might help with clinical application and acceptance of MR identification. These concepts complement the preceding two sections' collection and analytical integrated concept. T. Vercauteren, $\mathrm{M}$. Unberath, N. Padoy and N. Navab's fifth and final participation in this segment, "Portrait Renovation: From Sparse to Analysis Techniques and Deep Learning," examines how spatial, data-driven methods, and deep learning have predisposed and will remain to affect image restoration across mechanisms.

The [14] discuss existing fundamental methods and explain recent work targeted at interpreting what machine learning algorithms are really doing in this academically sound article. Model-based and data-driven image reconstruction approaches have been significantly impacted by sparse and reduced computational bundles, according to the authors. By looking at the chronology of video processing, they show how video quality is driving the creative process toward the cutting-edge approaches that are presently available. Sparsity has the largest effect on MR scan timings, whereas it has the biggest impact on CT patient dosage. Continuing to the ultrasonography subject, we observe that both contributions [15] and [16] illustrate how patchy and low-rank systems may reconstruct mixed frequency spectrum employing distinct constraints at the same time. Both articles include formal low-rank structures, like the L+S dissection, and also modern approaches [17]. For example, source separation may be used to remove unwanted interference or to separate physiological and functional data. In addition, a number of writers discuss current developments in the application of deep learning algorithms to image reconstruction in general [18]. The CNN cost and plug-and-play paradigm is a notably intriguing family of common hybrid domains methods [19]. Eventually, the researchers state that the next century imaging methods will use studying in all attributes of the image acquisition, discovering to maximize the great model for effective and efficient restoration and predictive modeling, such as concepts of categorization, fragmentation, and disorder object recognition, which is similar to the past articles in this paragraph [20].

\section{CROSS-CUTTING THEME}

The major purpose of the reported study was to expand on the authors' previous research and analyze the classification contribution of different algorithms. This is in the job of classifying tumours as malignant or benign, which is built utilizing human-engineered based biometric characteristics and two domain adaptation variants. These characteristics may be derived from pre-trained neurons or after a neural network has been fine-tuned for a particular categorization job. Employing dynamic contrast enhanced (DCE) Imaging techniques of human breasts, four distinct related fusion algorithms were investigated. "Wireless Capsule Endoscope: A Novel Instrument for Cancer Screening inside the Colorectal with Deep Learning-Based Lesion Detection," combines picture collection and image processing for application in screening mammography using wireless capsule endoscopy (WCE). In this study, machine learning or deep learning algorithms are being designed to aid in computerized polyp identification and research, which will improve diagnostics performance and reliability of this operation, which is a vital tool in the clinic.

WCE offers for straight inspecting of the complete colon without causing pain to the patient, however manual evaluation is time consuming. Computing approaches for automated polyp assessment have a lot of promise, and the adoption of deep learning algorithms for these jobs is gaining popularity and promises to improve performance and speed efficiency in the future. Convolutional neural networks (CNNs) are employed in the majority of these techniques, although machine learning algorithms and GANs have recently been used to identify polyp bounding boxes and transfer attributes learned from realistic photographs to be applied in melanoma feature extraction and classification studies. In table I of this paper, the researchers do an excellent job of laying out the range of computational intelligence methods used to identify WCE polyps, which could act as a blueprint for future research in this area. The $5^{\text {th }}$ and final research in this part, $\mathrm{T}$. Vercauteren, M. Unberath, N. Padoy and N. Navab in [16] "CAI4CAI: The Development of Contextual Intelligent Machines in Machine Vision Intervention," examines the application and rise of Ambient AI in Computer Aided Intervention strategies in the diagnostic imaging sector.

Incorporating a broad range of historical knowledge and real-time sensory information from experts, monitors, and actuators, as well as knowing how to develop a representations of the surgery or treatment among a mixed human community of performers, are the primary challenges in this area. The authors also show how to construct prophylactic and 
treatment procedures, as well as associated perceptive shared control mechanisms for online volatility in the OR or IR suite, work tasks that are critical for developing consistent and effective interventions tactics. Much of this requires the integration of many types of treatment data, such as photographs for treatments or surgery assistance, prompting the coining of the term "surgical computing sciences." We notice a few common themes as we read through the 10 contributions to our Theme Issue. To begin, most investigations' research made the critical observation that data-driven deep learning could be able to consolidate the often modularized design of imaging techniques. The procedures that enable ultrasounds to be used to monitor sickness may now become more linked, and we may see comprehensive end-to-end new designs that are task-based to optimize efficiency.

\section{CONCLUSION}

Machine learning techniques may be used to recognize patterns in many kinds of digital data, such as pictures, texts, and videos. Finding patterns allows you to categorize your findings and make more educated decisions. Pattern recognition is a powerful tool for completely automating and solving complex analytical issues. The ability to see the human body using a variety of modalities has transformed medicine in recent decades and continues to advance at a fast rate. At a variety of spatiotemporal scales, previously undiscovered knowledge about biology and illness is being revealed more than ever before. While findings and acceptance of methods for computational and quantitative image analysis have trailed behind image capture technologies, these fields have seen a surge of interest and effort in recent times. This new issue attempts to identify and emphasize some of the "current" newer concepts in biological applications and analysis, with the goal of shedding light on where the discipline could go in the next decades, with an emphasis about where electrical designers have been engaged and might have the greatest effect. Among these topics are picture capture mechanics, image/signal analysis, and laser scanning, which include object identification and recognition. The focus of the issue was on themes which thread across most of this work: Firstly, engineers and software developers recognized that when examining medical images using image-based linear spaces, the data available is generally minimal. This comment has had a big influence on a lot of individuals, and it shows up in a lot of the tales we've collected here. Secondly, since ultrasound imaging is one of the most significant sources of "huge data," data-oriented machine learning approaches (such as pattern recognition) are gaining wide acceptance owing to their high efficacy.

\section{References}

[1]. D. Brown, C. Dickson, P. Duncan, F. Al-Attili and V. Stone, "Interaction between nanoparticles and cytokine proteins: impact on protein and particle functionality", Nanotechnology, vol. 21, no. 21, p. 215104, 2010. Doi:10.1088/0957-4484/21/21/215104.

[2]. R. Damadian, "5606970 Multiple patient scanning on a magnetic resonance imaging apparatus", Magnetic Resonance Imaging, vol. 15, no. 5, p. XIII, 1997. Doi:10.1016/s0730-725x(97)89749-4.

[3]. C. Keskin and L. Akarun, "STARS: Sign tracking and recognition system using input-output HMMs", Pattern Recognition Letters, vol. 30, no. 12, pp. 1086-1095, 2009. Doi:10.1016/j.patrec.2009.03.016.

[4]. S. Koinuma, Y. Umesono, K. Watanabe and K. Agata, "The expression of planarian brain factor homologs, DjFoxG and DjFoxD", Gene Expression Patterns, vol. 3, no. 1, pp. 21-27, 2003. Doi:10.1016/s1567-133x(02)00097-2.

[5]. P. Mahajan, "Applications of Pattern Recognition Algorithm in Health and Medicine: A", International Journal Of Engineering And Computer Science, 2016. Doi:10.18535/ijecs/v5i5.41.

[6]. P. Hanrath, "Imaging Techniques: Transoesophageal Echo-Doppler in cardiology", Heart, vol. 86, no. 5, pp. 586-592, 2001. Doi:10.1136/heart.86.5.586.

[7]. Y. Wang, E. Li, S. Cherry and G. Wang, "Total-Body PET Kinetic Modeling and Potential Opportunities Using Deep Learning", PET Clinics, vol. 16, no. 4, pp. 613-625, 2021. Doi:10.1016/j.cpet.2021.06.009.

[8]. A. Kaur and I. Kaur, "Empirical Evaluation of Machine Learning Algorithms for Fault Prediction", Lecture Notes on Software Engineering, pp. 176-180, 2014. Doi:10.7763/lnse.2014.v2.118.

[9]. L. Dorst and R. Van den Boomgaard, "Morphological signal processing and the slope transform", Signal Processing, vol. 38, no. 1, pp. 79-98, 1994. Doi:10.1016/0165-1684(94)90058-2

[10]. S. Ravishankar and Y. Bresler, "Learning Sparsifying Transforms", IEEE Transactions on Signal Processing, vol. 61, no. 5, pp. 1072-1086, 2013. Doi:10.1109/tsp.2012.2226449.

[11]. A. Haldorai and A. Ramu, "Canonical Correlation Analysis Based Hyper Basis Feedforward Neural Network Classification for Urban Sustainability," Neural Processing Letters, Aug. 2020. doi:10.1007/s11063-020-10327-3

[12]. D. Devikanniga, A. Ramu, and A. Haldorai, "Efficient Diagnosis of Liver Disease using Support Vector Machine Optimized with Crows Search Algorithm," EAI Endorsed Transactions on Energy Web, p. 164177, Jul. 2018. doi:10.4108/eai.13-7-2018.164177

[13]. H. Anandakumar and K. Umamaheswari, "A bio-inspired swarm intelligence technique for social aware cognitive radio handovers," Computers \& Electrical Engineering, vol. 71, pp. 925-937, Oct. 2018. doi:10.1016/j.compeleceng.2017.09.016

[14]. R. Arulmurugan and H. Anandakumar, "Early Detection of Lung Cancer Using Wavelet Feature Descriptor and Feed Forward Back Propagation Neural Networks Classifier," Lecture Notes in Computational Vision and Biomechanics, pp. 103-110, 2018. doi:10.1007/978-3319-71767-8_9

[15]. Haldorai, A. Ramu, and S. Murugan, "Social Aware Cognitive Radio Networks," Social Network Analytics for Contemporary Business Organizations, pp. 188-202. doi:10.4018/978-1-5225-5097-6.ch010

[16]. R. Arulmurugan and H. Anandakumar, "Region-based seed point cell segmentation and detection for biomedical image analysis," International Journal of Biomedical Engineering and Technology, vol. 27, no. 4, p. 273, 2018.

[17]. M. Suganya and H. Anandakumar, "Handover based spectrum allocation in cognitive radio networks," 2013 International Conference on Green Computing, Communication and Conservation of Energy (ICGCE), Dec. 2013.doi:10.1109/icgce.2013.6823431. doi:10.4018/978-15225-5246-8.ch012

[18]. Haldorai and A. Ramu, "An Intelligent-Based Wavelet Classifier for Accurate Prediction of Breast Cancer," Intelligent Multidimensional Data and Image Processing, pp. 306-319.

[19]. S, D., \& H, A. (2019). AODV Route Discovery and Route Maintenance in MANETs. 2019 5th International Conference on Advanced Computing \& Communication Systems (ICACCS). doi:10.1109/icaccs.2019.8728456

[20]. T. Vercauteren, M. Unberath, N. Padoy and N. Navab, "CAI4CAI: The Rise of Contextual Artificial Intelligence in Computer-Assisted Interventions", Proceedings of the IEEE, vol. 108, no. 1, pp. 198-214, 2020. Doi:10.1109/jproc.2019.2946993. 